\title{
Telomere identity crisis
}

\author{
Bridget L. Baumgartner ${ }^{1,2}$ and Vicki Lundblad ${ }^{1,3}$ \\ ${ }^{1}$ Salk Institute for Biological Research, La Jolla, California 92037, USA; ${ }^{2}$ Interdepartmental Program in Cell and Molecular \\ Biology, Baylor College of Medicine, Houston, Texas 77030, USA
}

Cells are designed to be intolerant of breaks in DNA, yet it is critical that cells do not identify the ends of linear chromosomes, called telomeres, as damaged DNA ends. Telomeres therefore must somehow prevent the recognition and subsequent repair of chromosome ends as double-strand breaks (DSBs), although how this is achieved is poorly understood. Chromosomal breaks can occur as a result of ionizing radiation, DNA replication across nicked DNA, or as intermediates of recombination. Whether these breaks are induced by the cell-for example, due to $\mathrm{V}(\mathrm{D}) \mathrm{J}$ recombination during lymphocyte development—or arise as a consequence of spontaneous damage, it is imperative that such lesions be repaired in order to prevent genomic rearrangements or even outright chromosome loss (Bassing and Alt 2004).

The cell avoids such deleterious consequences by mounting a response, called the DNA damage checkpoint, which pauses the cell cycle, thereby permitting the efficient recruitment of a highly conserved set of proteins to the break (Zhou and Elledge 2000; Nyberg et al. 2002). In the budding yeast Saccharomyces cerevisiae, this process has been studied extensively using an experimental system that allows the creation of a single DSB at high frequency at a defined site, through the action of a sequence-specific endonuclease, $\mathrm{HO}$ (Rudin and Haber 1988). The DNA damage checkpoint is alerted to the presence of the HO-generated break by recruitment of sensor proteins, such as Tell/ATM and Mec1/ATR, to the site of damage (Lisby et al. 2004; Garber et al. 2005). These kinases activate downstream effector proteins, Rad53/CHK2, Rad9, and Dun1, which in turn facilitate the activation of repair proteins. Repair of DSBs requires the recognition of these broken ends by the Ku70/80 heterodimer and the Mre11-Rad50-Xrs2 complex (MRX, or MRN in humans). The cell then has a choice between two pathways for DNA repair. If DSBs are repaired through the nonhomologous end-joining pathway (NHEJ), DNA ligase 4 is brought in to seal the two ends together. If the repair is accomplished by homologous recombination, the DSB is first processed by an exonuclease to reveal a single-stranded 3 ' overhang, which subsequently initiates recombination with homologous

${ }^{3}$ Corresponding author.

EMAIL lundblad@salk.edu; FAX (858) 457-4765.

Article and publication are at http://www.genesdev.org/cgi/doi/10.1101/ gad.1373205. sequences present in the genome. Functional DNA damage checkpoints also act to inhibit cell cycle progression in the presence of damaged DNA. Even a single DSB is sufficient to cause the cell cycle to arrest until repair is completed (Sandell and Zakian 1993), thereby ensuring that cells do not progress through mitosis until the integrity of the genome has been restored.

Despite the presence of a highly efficient machine for the recognition of DSBs, the ends of linear chromosomes are natural DNA termini that must somehow be masked from triggering the DNA damage checkpoint and subsequent repair events. This unique property of telomeres is owed to the sequence and structure of the telomere DNA itself, as well as to the proteins that localize to chromosome ends (Blackburn 2001; Smogorzewska and de Lange 2004). In most eukaryotes, telomeres are composed of tandem G-rich repeats that terminate with a $3^{\prime}$ singlestranded overhang of the G-rich strand, often referred to as a G-tail. Disruption of this G-tail structure, due to either loss of the G-tail itself or exposure of the C-strand to nucleolytic attack, is a lethal event for the cell. Thus, cells have developed a dynamic protein assembly that maintains a telomere "cap." In budding yeast, this cap depends in part on the essential single-strand telomerebinding protein $\mathrm{Cdc13}$, along with several Cdc13-interacting factors. Loss of the Cdc13 complex exposes yeast telomeres to massive resection of the C-strand, with the resulting 20-30-kb region of exposed single-stranded DNA provoking arrest of the cell cycle (Weinert et al. 1994; Garvik et al. 1995; Booth et al. 2001). A second protein, Rap1, which is bound to duplex telomeric repeats, may aid in protection of the other strand of the telomere, the G-strand overhang: Depleting cells of Rap1 causes telomere fusions that are mediated through the NHEJ pathway (Pardo and Marcand 2005). Mammalian cells also possess a mechanism to protect the vulnerable G-tail, which similarly relies on a duplex telomere DNA-binding factor called TRF2. In TRF2-deficient cells, the integrity of the terminal G-strand overhang is disrupted, resulting in high frequencies of end-to-end fusions (van Steensel et al. 1998; Celli and de Lange 2005). This TRF2-mediated telomere capping activity may be aided by the ability of telomeres to fold into a looped structure, called the t-loop, wherein the $3^{\prime}$ overhang invades the duplex region of telomere repeats (Griffith et al. 1999).

Not unexpectedly, normal telomeres rarely interact 
with DSBs. However, loss of telomere repeats can lead to "repair" of chromosome ends, through either recombination (Lundblad and Blackburn 1993; Bryan et al. 1995) or end-to-end fusions (Smogorzewska et al. 2002). The latter situation creates dicentric chromosomes, which are unstable in dividing cells. Dysfunctional telomeres can be created experimentally, through genetic manipulation of telomere-associated components or by altering the sequence of the telomere repeats themselves. Human cells, in which telomerase expression has been down-regulated, can also accumulate eroded telomeres as a consequence of continual cell division. In both cases, critically shortened telomeres trigger a damage response that prevents the cells from dividing in the absence of intact chromosome ends. Cells can, however, escape this block to proliferation (albeit at very low frequencies) and continue to divide in the absence of functional telomeres, but at a price: Such "escapees" display massive levels of chromosomal rearrangements, illustrating the importance of telomeres for genome stability.

Given all this, it is tempting to assume that telomeres prevent components of DNA damage-responsive pathways from interacting with the ends of chromosomes. However, the situation is more complicated, as many of the genes required for DNA repair are also necessary for normal telomere maintenance (Maser and DePinho 2004). The intertwining of DSB repair and telomere maintenance extends to pathways involved in signaling damage, as well as to those responsible for the actual repair of damage. For example, the members of the NHEJ pathway are intimately involved in telomere maintenance. This is paradoxical considering it is the NHEJ pathway that causes the fusion of chromosomes when telomeres become dysfunctional, and yet, the components of this pathway affect multiple facets of telomere metabolism. In yeast, the Ku heterodimer exhibits a robust association with telomeres and is required for capping, modulation of subtelomeric gene expression, and even telomere length regulation as the result of a direct interaction with the telomerase holoenzyme (Bertuch and Lundblad 2003). Likewise, deletion of any of the MRX subunits leads to telomere shortening (Boulton and Jackson 1998), possibly because telomere-specific proteins such as Cdc13 fail to be recruited to telomeres in the absence of MRX function (Diede and Gottschling

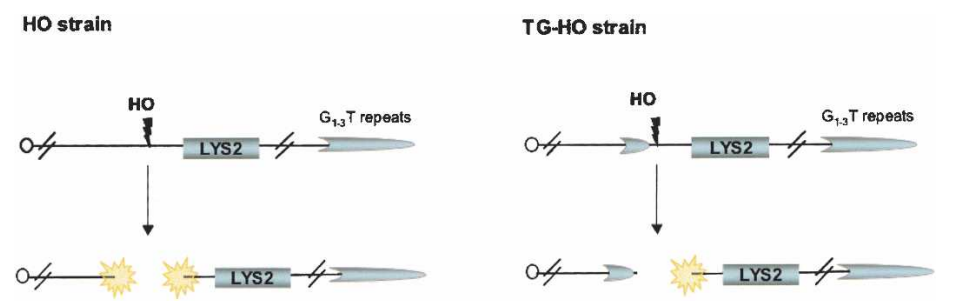

= DSB signal
2001; Takata et al. 2005). Furthermore, the sensor proteins Tell and Mec1 exhibit a reciprocal, and cell cycleregulated, association with telomeres in budding yeast (Takata et al. 2004). How the checkpoint activity of these two proteins is modulated, when they are localized at telomeres, is unknown.

Although this area of telomere biology is under intense investigation, little is known about the mechanisms that normal telomeres employ to effectively protect chromosome ends from DNA repair pathways. Work from the Weinert group, published in this issue of Genes \& Development (Michelson et al. 2005), provides new insights into this process, by reporting that telomere repeats are able to quench local DNA damage responses, thereby conferring what the authors dub an "anticheckpoint." Their experimental approach exploited a previously developed system to induce de novo telomere formation at a newly created DNA end (Diede and Gottschling 1999): An inducible site-specific endonuclease, called HO, is used to generate a DSB immediately downstream of an internal tract of yeast telomere repeats (Fig. 1). Because these experiments are performed in a haploid yeast strain, repair of the DSB by conventional homologous recombination is a rare event. Instead, the presence of the telomeric seed sequence is able to direct the repair of this DNA end as a telomere, which allows the cell to survive the otherwise lethal consequences of this irreparable DSB. Formation of a de novo telomere at this newly exposed terminus depends on the same factors that mediate normal telomere function, such as MRX activity, binding of the Cdc13 complex, and subsequent extension of the telomere repeats by telomerase (Diede and Gottschling 1999, 2001).

Although telomerase and other telomere-binding proteins can convert one of the two products of this HOgenerated break into a functional telomere, what about the other DNA end created by this break? Michelson et al. (2005) discovered that although the nontelomeric product of the cleavage reaction (i.e., the end of the distal portion of the chromosome) (Fig. 1) was still capable of invoking an initial DNA damage response, the extent of this response was affected by the proximity of the DSB to the new telomere. As expected from previous studies, induction of the DSB in either strain-the TG-HO strain, containing a telomere seed sequence adjacent to the

Figure 1. The experimental system used to detect the anticheckpoint activity relies on a modified chromosome that has been engineered to contain a single recognition site for the site-specific endonuclease, HO. Regulated induction of $\mathrm{HO}$ expression causes a DSB at this site at a high frequency in a population of cells. In the HO control strain, the DSB is recognized by the DNA damage response on both sides of the break. The TG-HO strain has a tract of yeast $\mathrm{G}_{1-3} \mathrm{~T}$ telomere repeats adjacent to the $\mathrm{HO}$ cut site, such that generation of a DSB in this strain creates a DSB on one end and a substrate for de novo telomere formation on the other end. The DSB is able to signal a DNA damage response, whereas the exposed telomeric sequences are treated as though they were natural telomeres. 
break, or the control HO strain-was able to cause a DNA damage response and mitotic arrest with similar initial kinetics. However, while the control HO strain remained arrested for up to $8 \mathrm{~h}$, the TG-HO strain was able to overcome its mitotic arrest and resume the cell cycle after only $1-2 \mathrm{~h}$. This dramatic difference in the duration of the DNA damage responses in these two strains suggests that the telomere seed sequence, present on one of the newly exposed DNA ends, influences the DNA damage response sent by the other DNA end that lacks any telomeric sequences

What is the essence of this abridged arrest? The arrest itself, in both the control HO strain and the TG-HO strain, is dependent on the DNA damage response, as deletion of required genes, such as MEC1, abolished the cell cycle delay in both strains. Thus, the initial response to the DSB is the same, regardless of the presence of adjacent telomere sequences. Repair of the non-telomere-containing DNA end was also unaffected in TG-HO strains, as measured by the kinetics of resection of the distal arm. One possible mechanism for the attenuation of this arrest is that the telomere repeats allow the cells to adapt to the checkpoint more quickly. Adaptation is a phenomenon observed in yeast, where cells are sometimes able to overcome the DNA damage response and reenter the cell cycle without first repairing the DNA damage (Toczyski et al. 1997). However, defects in genes known to be required for adaptation had no effect on the abridged arrest observed in the TG-HO strain. Weinert's group (Michelson et al. 2005) therefore turned their attention to the duration of the DNA damage response. This response is a kinase cascade that can amplify the signal from a single DSB and ultimately halt the progression of the cell cycle. The activity of the response can be monitored by measuring the activity of the pathway's kinases, and by detecting phosphorylation of the target substrates. Using these techniques, the activation of the pathway in response to a DSB occurred with the same kinetics in both TG-HO and HO strains, consistent with the observation that both strains arrested their cell cycles in the same amount of time. However, the activity of the pathway did not persist in the TG-HO strain: The DNA damage response was attenuated within $3 \mathrm{~h}$, as measured by Rad53 kinase activity, in this strain. In contrast, Rad53 activity continued to increase for up to $5 \mathrm{~h}$ in the control strain. Thus, the DNA damage response pathway is actively inhibited somewhere upstream of Rad53 activation, when telomeric sequences are present adjacent to the break.

Thus, telomere sequences can confer an anticheckpoint activity on nontelomeric DNA ends. This anticheckpoint can even effectively suppress the DNA damage response stemming from breaks that occur $>0.5$ from a telomere repeat sequences. However, the effect is only exerted in cis: Breaks on another chromosome arm were not subjected to this anticheckpoint mechanism. Perhaps the telomere creates a local protein environment that acts like a protective shell to shield the chromosome ends from the activity of DNA repair factors.

The nature of the anticheckpoint element is open to

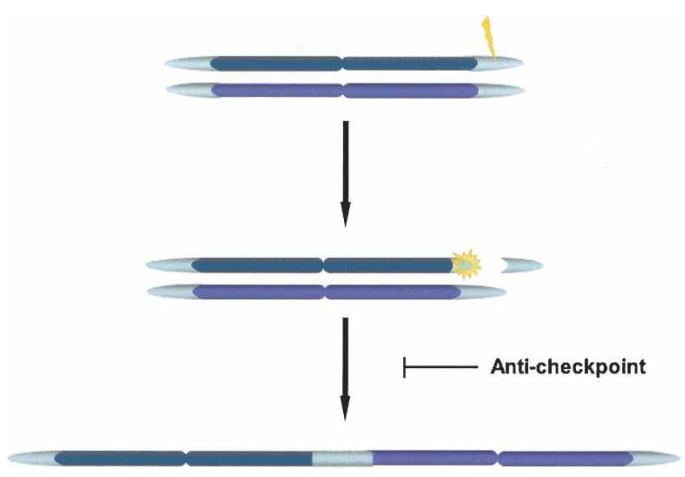

Figure 2. One possible role for the anticheckpoint activity is to prevent the accidental fusion of telomeres when they are in a vulnerable state, as a result of rare DNA breaks occurring within the telomeric repeat array. Such telomere-localized breaks could arise due to improper resolution of intermediates of DNA metabolism, such as the unintended byproduct of intrachromatid deletions that restore elongated telomeres to wildtype lengths (called Telomere Rapid Deletion) (Li and Lustig 1996) or as a result of DNA replication across nicks or gaps that have been postulated to exist in the C-strand of the telomere (Szostak and Blackburn 1982).

speculation, although there are several compelling candidates in both the yeast and mammalian systems. The most obvious candidate in human cells is TRF2, as TRF2 has already been shown to bind to, and inhibit, the activation of the ATM kinase (Karlseder et al. 2004). Although budding yeast has no ortholog of TRF2, the anticheckpoint function may be conserved in a telomerebinding protein that is required for capping function, such as Cdc13, which would be consistent with the suggestion by Michelson et al. (2005) that the anticheckpoint protein will be an essential factor. Alternatively, the anticheckpoint may be redundant with other mechanisms that prevent chromosome fusions. It is also not clear whether the anticheckpoint activity contributes to normal telomere function in every cell cycle or only responds to telomere dysfunction. Weinert and colleagues (Michelson et al. 2005) propose that "maturation" of a DNA end into a functional telomere relies on recruitment of protein factors, which includes the anticheckpoint activity. Alternatively, the anticheckpoint may be required to inactivate the DNA damage response only when it is likely to do more harm than good. For example, if a DSB were to occur in a subtelomeric region, it would be better for the cell to lose the broken fragment (as telomeres are not often near essential genes) than to mistakenly repair it by fusion to the end of a sister chromatid (Fig. 2). In this ironic situation, an anticheckpoint system would be critical to prevent damage by the DNA repair pathway.

\section{References}

Bassing, C.H. and Alt, F.W. 2004. The cellular response to general and programmed DNA double strand breaks. DNA Repair 3: 781-796. 
Bertuch, A.A. and Lundblad, V. 2003. Which end: Dissecting $\mathrm{Ku}$ 's function at telomeres and double-strand breaks. Genes \& Dev. 17: 2347-2350.

Blackburn, E.H. 2001. Switching and signaling at the telomere. Cell 106: 661-673.

Booth, C., Griffith, E., Brady, G., and Lydall, D. 2001. Quantitative amplification of single-stranded DNA (QAOS) demonstrates that $c d c 13-1$ mutants generate ssDNA in a telomere to centromere direction. Nucleic Acids Res. 20: 4414-4422.

Boulton, S.J. and Jackson, S.P. 1998. Components of the Kudependent non-homologous end-joining pathway are involved in telomeric length maintenance and telomeric silencing. EMBO J. 17: 1819-1828.

Bryan, T.M., Englezou, A., Gupta, J., Bacchetti S., and Reddel, R.R. 1995. Telomere elongation in immortal human cells without detectable telomerase activity. EMBO J. 14: 42404248.

Celli, G.B. and de Lange, T. 2005. DNA processing is not required for ATM-mediated telomere damage response after TRF2 deletion. Nat. Cell Biol. 7: 712-718.

Diede, S.J. and Gottschling, D.E. 1999. Telomerase-mediated telomere addition in vivo requires DNA primase and DNA polymerases $\alpha$ and $\delta$. Cell 99: 723-733.

- 2001. Exonuclease activity is required for sequence addition and Cdc13p loading at a de novo telomere. Curr. Biol 11: 1336-1340.

Garber, P.M., Vidanes, G.M., and Toczyski, D.P. 2005. Damage in transition. Trends Biochem. Sci. 30: 63-66.

Garvik, B., Carson, M., and Hartwell, L. 1995. Single-stranded DNA arising at telomeres in $c d c 13$ mutants may constitute a specific signal for the RAD9 checkpoint. Mol. Cell. Biol. 15: 6128-6138.

Griffith, J.D., Comeau, L., Rosenfield, S., Stansel, R.M., Bianchi, A., Moss, H., and de Lange, T. 1999. Mammalian telomeres end in a large duplex loop. Cell 97: 503-514.

Karlseder, J., Hoke, K., Mirzoeva, O.K., Bakkenist, C., Kastan, M.B., Petrini, J.H., and de Lange, T. 2004. The telomeric protein TRF2 binds the ATM kinase and can inhibit the ATM-dependent DNA damage response. PLOS Biol. 2: E240.

Li, B. and Lustig, A.J. 1996. A novel mechanism for telomere size control in Saccharomyces cerevisiae. Genes \& Dev. 10: $1310-1326$.

Lisby, M., Barlow, J.H., Burgess, R.C., and Rothstein, R. 2004. Choreography of the DNA damage response: Spatiotemporal relationships among checkpoint and repair proteins. Cell 118: 699-713.

Lundblad, V. and Blackburn, E.H. 1993. An alternative pathway for yeast telomere maintenance rescues est $1^{-}$senescence. Cell 73: 347-360.

Maser, R.S. and DePinho, R.A. 2004. Telomeres and the DNA damage response: Why the fox is guarding the henhouse. DNA Repair (Amst) 3: 979-988.

Michelson, R.J., Rosenstein, S., and Weinert, T. 2005. A telomeric repeat sequence adjacent to a DNA double-strand break produces an anticheckpoint. Genes \& Dev. (this issue).

Nyberg, K.A., Michelson, R.J., Putnam, C.W., and Weinert, T.A. 2002. Toward maintaining the genome: DNA damage and replication checkpoints. Annu. Rev. Genet. 36: 617-656.

Pardo, B. and Marcand, S. 2005. Rap1 prevents telomere fusions by nonhomologous end joining. EMBO J. 24: 3117-3127.

Rudin, N. and Haber, J.E. 1988. Efficient repair of HO-induced chromosomal breaks in Saccharomyces cerevisiae by recombination between flanking homologous sequences. Mol. Cell. Biol. 8: 3918-3928.

Sandell, L.L. and Zakian, V.A. 1993. Loss of a yeast telomere: Arrest, recovery, and chromosome loss. Cell 75: 729-739.
Smogorzewska, A. and de Lange, T. 2004. Regulation of telomerase by telomeric proteins. Annu. Rev. Biochem. 73: 177208.

Smogorzewska, A., Karlseder, J., Holtgreve-Grez, H., Jauch, A., and de Lange, T. 2002. DNA ligase IV-dependent NHEJ of deprotected mammalian telomeres in G1 and G2. Curr. Biol. 12: $1635-1644$.

Szostak, J.W. and Blackburn, E.H. 1982. Cloning yeast telomeres on linear plasmid vectors. Cell 29: 245-255.

Takata, H., Kanoh, Y., Gunge, N., Shirahige, K., and Matsuura, A. 2004. Reciprocal association of the budding yeast ATMrelated proteins Tell and Mec1 with telomeres in vivo. Mol. Cell 14: 515-522.

Takata, H., Tanaka, Y., and Matsuura, A. 2005. Late S phasespecific recruitment of Mre11 complex triggers hierarchical assembly of telomere replication proteins in Saccharomyces cerevisiae. Mol. Cell 17: 573-583.

Toczyski, D.P., Galgoczy, D.J., and Hartwell, L.H. 1997. CDC5 and CKII control adaptation to the yeast DNA damage checkpoint. Cell 90: 1097-1106.

van Steensel, B., Smogorzewska, A., and de Lange, T. 1998. TRF2 protects human telomeres from end-to-end fusions. Cell 92: 401-413.

Weinert, T.A., Kiser, G.L., and Hartwell, L.H. 1994. Mitotic checkpoint genes in budding yeast and the dependence of mitosis on DNA replication and repair. Genes \& Dev. 8: 652-665.

Zhou, B.B. and Elledge, S.J. 2000. The DNA damage response: Putting checkpoints in perspective. Nature 408: 433-439. 


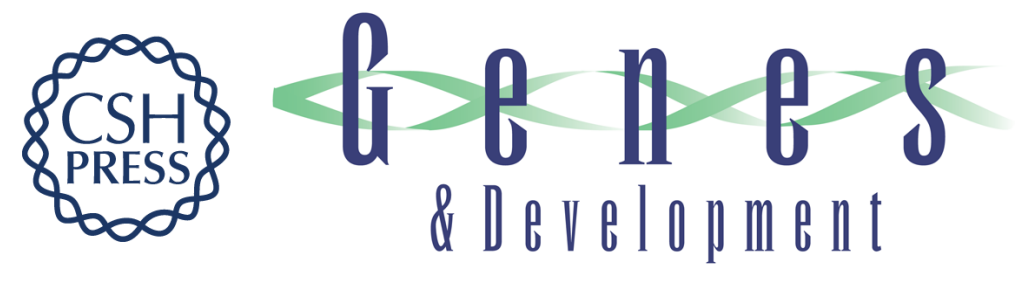

\section{Telomere identity crisis}

Bridget L. Baumgartner and Vicki Lundblad

Genes Dev. 2005, 19:

Access the most recent version at doi:10.1101/gad.1373205

References This article cites 28 articles, 7 of which can be accessed free at: http://genesdev.cshlp.org/content/19/21/2522.full.html\#ref-list-1

License

Email Alerting Receive free email alerts when new articles cite this article - sign up in the box at the top Service right corner of the article or click here.

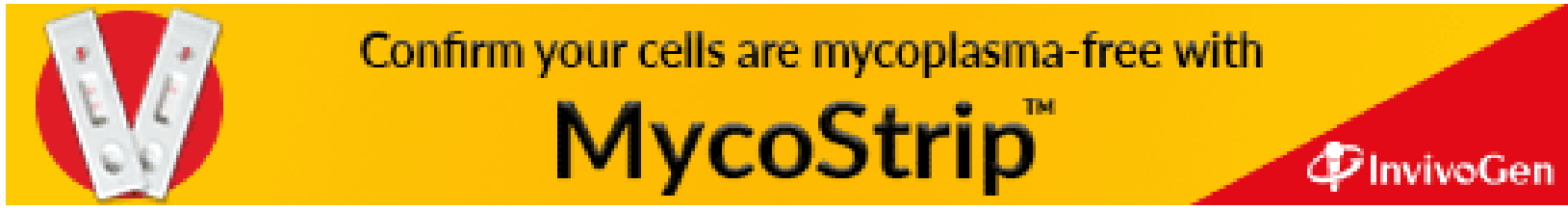

